School of Public Health China Medical University; No.77

Puhe Road, Shenyang North New Area, Shenyang, Liaoning

Province 110122, China.

Email:ynma@cmu.edu.cn

Zhang and He these authors contributed equally as first co-authors. Yuan and Ma these authors contributed equally as senior co-

authors.

ORCID

Yanan Ma iD https://orcid.org/0000-0003-0385-8929

\section{REFERENCES}

1. Mallol J, Crane J, Von Mutius E, et al. The international study of asthma and allergies in childhood (ISAAC) phase three: a global synthesis[J]. Allergol Immunopathol. 2013;41(2):73-85.

2. Suk WA, Ahanchian $\mathrm{H}$, Asante KA, et al. Environmental pollution: an under-recognized threat to children's health, especially in low- and middle-income countries. Environ Health Perspect. 2016;124(3):A41-A45.

3. Huang S, Garshick E, Weschler LB, et al. Home environmental and lifestyle factors associated with asthma, rhinitis and wheeze in children in Beijing, China. Environ Pollut. 1987;2020(256):113426.
4. Colton MD, Laurent JG, Macnaughton P, et al. Health benefits of green public housing: associations with asthma morbidity and building-related symptoms. Am J Public Health. 2015;105(12):2482-2489.

5. Ranathunga N, Perera P, Nandasena S, et al. Effect of household air pollution due to solid fuel combustion on childhood respiratory diseases in a semi urban population in Sri Lanka. BMC Pediatr. 2019;19(1):306.

6. Liang $X$, Huang $T$, Lin S, et al. Chemical composition and source apportionment of $\mathrm{PM}(1)$ and $\mathrm{PM}(2.5)$ in a national coal chemical industrial base of the golden energy triangle, Northwest China[J]. Sci Total Environ. 2019;659:188-199.

7. Qiu G, Song R, He S. The aggravation of urban air quality deterioration due to urbanization, transportation and economic development - Panel models with marginal effect analyses across China[J]. Sci Total Environ. 2019;651(Pt 1):1114-1125.

8. Brandt EB, Myers JM, Ryan $\mathrm{PH}$, et al. Air pollution and allergic diseases[J]. Curr Opin Pediatr. 2015;27(6):724-735.

9. Tran VV, Park D, Lee YC. Indoor air pollution, related human diseases, and recent trends in the control and improvement of indoor air quality[J]. Int J Environ Res Public Health. 2020;17(8):2927.

\section{SUPPORTING INFORMATION}

Additional supporting information may be found online in the Supporting Information section.

\title{
Brain activation after nasal histamine provocation in house dust mite allergic rhinitis patients
}

To the Editor,

The nasal mucosa is armed with a complex nervous system of sensory, sympathetic, and parasympathetic nerves, allowing swift defensive responses to physical and chemical stimuli. In allergic rhinitis (AR) patients, nasal allergen deposition leads to mast cell activation with release of allergic mediators such as histamine. Apart from its direct effects on the surrounding tissue, histamine also activates sensory nerve endings giving rise to symptoms like sneezing, rhinorrhea, and/or congestion. ${ }^{1}$ Activated nasal sensory nerves transmit action potentials to their cell bodies in the trigeminal ganglion and further to the midbrain where secondary synapses lead to the generation of central reflex signals. Despite activation of neural pathways in $\mathrm{AR},{ }^{2}$ it is not known which particular regions in the brain are activated by different nasal stimuli. Clinical studies using positron emission tomography scans indicate that there is no isolated itch center in the brain but that different cortical centers are involved in the processing of itch. ${ }^{3,4}$ Activation of the anterior cingulate cortex (ACC), the supplementary motor area (SMA), and the inferior parietal lobe partly explains the connection between itching and the related reflex of scratching. ${ }^{4}$ Using functional magnetic resonance imaging (fMRI), the activation of the superior temporal gyrus, insula, and nucleus caudate following painful intranasal trigeminal stimulation has been shown. ${ }^{5}$ When asthmatic patients are challenged with methacholine or allergens, activity in ACC and insula was associated with markers of bronchial inflammation and obstruction. ${ }^{6}$

To fill the abovementioned knowledge gap, a prospective, single-blind, cross-over study was designed to investigate brain responses to nasal histamine provocation in healthy volunteers and AR patients.

In this proof-of-concept study, 8 house dust mite (HDM) AR patients and 7 non-allergic healthy controls $(\mathrm{HC})$ were recruited at the outpatient clinic for Otorhinolaryngology of University Hospitals Leuven. $\mathrm{HC}$ had a negative skin prick test, no nasal symptoms, and had no nasal anatomic abnormalities or rhinosinusitis as confirmed by nasal endoscopy. AR patients had a positive skin prick test for $D$. pteronyssinus and/or D. farinae $>5 \mathrm{~mm}$. Two out of 8 AR patients

This is an open access article under the terms of the Creative Commons Attribution-NonCommercial License, which permits use, distribution and reproduction in any medium, provided the original work is properly cited and is not used for commercial purposes.

(c) 2020 The Authors. Allergy published by European Academy of Allergy and Clinical Immunology and John Wiley \& Sons Ltd. 


\begin{tabular}{|c|c|c|c|c|}
\hline $\begin{array}{l}\text { MNI coordinates of } \\
\text { local maximum ( } x \text { y } z \text { ) }\end{array}$ & $\begin{array}{l}\text { cluster } \\
\text { extent }(k)\end{array}$ & $\begin{array}{l}\text { F-value } \\
\text { local } \\
\text { maximum }\end{array}$ & anatomic localization & $\begin{array}{l}\text { direction of } \\
\text { difference }\end{array}$ \\
\hline$-60-168$ & 70 & 14.03 & $\begin{array}{l}\text { Left postcentral gyrus } \\
\text { left Rolandic operculum } \\
\text { left superior temporal } \\
\text { gyrus }\end{array}$ & $\mathrm{HC}>\mathrm{AR}$ \\
\hline $54-1-1$ & 47 & 9.33 & $\begin{array}{l}\text { Right posterior insula } \\
\text { right superior temporal } \\
\text { gyrus/pole }\end{array}$ & $\mathrm{HC}>\mathrm{AR}$ \\
\hline $36-7335$ & 14 & 9.20 & $\begin{array}{l}\text { Right middle occipital } \\
\text { gyrus }\end{array}$ & $A R>H C$ \\
\hline $60-3120$ & 20 & 9.15 & $\begin{array}{l}\text { Right superior temporal } \\
\text { gyrus } \\
\text { right supramarginal gyrus }\end{array}$ & $\mathrm{HC}>\mathrm{AR}$ \\
\hline$-30-64-40$ & 21 & 9.05 & $\begin{array}{l}\text { Left cerebellum (crus } 1 \\
\text { and } 2 \text { ) }\end{array}$ & $A R>H C$ \\
\hline$-27-411$ & 20 & 9.01 & $\begin{array}{l}\text { Left mid insula } \\
\text { left putamen }\end{array}$ & $\mathrm{HC}>\mathrm{AR}$ \\
\hline $029-13$ & 15 & 7.68 & $\begin{array}{l}\text { medial orbital gyrus } \\
\text { gyrus rectus }\end{array}$ & $\mathrm{HC}>\mathrm{AR}$ \\
\hline 2788 & 13 & 7.54 & Right putamen & $\mathrm{HC}>\mathrm{AR}$ \\
\hline 39565 & 10 & 7.13 & $\begin{array}{l}\text { Right superior/middle } \\
\text { frontal gyrus }\end{array}$ & $A R>H C$ \\
\hline $63-1311$ & 12 & 6.89 & $\begin{array}{l}\text { Right Rolandic } \\
\text { operculum } \\
\text { right superior temporal } \\
\text { gyrus }\end{array}$ & $\mathrm{HC}>\mathrm{AR}$ \\
\hline $368-16$ & 10 & 6.80 & Right anterior insula & $A R>H C$ \\
\hline
\end{tabular}

TABLE 1 Overview of brain regions in which the response to histamine (versus saline as a control condition) differs between allergic rhinitis patients and healthy controls

Note: All local maxima $\mathrm{p}_{\mathrm{FWE}}<0.001$, all clusters $\mathrm{p}_{\mathrm{FWE}}<0.001$.

Abbreviations: AR, allergic rhinitis; FWE, family-wise error; HC, healthy controls; MNI, Montreal Neurological Institute.

were mono-sensitized to HDM, and the other 6 AR patients were poly-sensitized with sensitizations to cat $(n=6), \operatorname{dog}(n=5)$, tree pollen $(n=4)$, or grass pollen $(n=5)$. Patients using nasal or oral steroid treatment $<6$ weeks or nasal or oral antihistamine treatment $<4$ weeks prior to the study were excluded, as well as those with past or ongoing immunotherapy for HDM, asthma, smoking, and clinical signs of rhinosinusitis or anatomic nasal deformities.

All study participants were between 18 and 50 years old and provided written informed consent. The study was approved by the local medical ethics committee of the University Hospitals Leuven (B322201215751).

$\mathrm{HC}$ and $\mathrm{AR}$ patients underwent a nasal provocation through a cannula placed under the nose with either nebulized sham solution (saline) or with histamine for 5 minutes while in a supine position in the MR scanner on 2 separate days with a minimum of 1 week in between and in a single-blinded and random order. An aerosol of $10 \mathrm{~mL}$ histamine $\mathrm{HCl}(16 \mathrm{mg} / \mathrm{mL})$ or $10 \mathrm{~mL}$ saline was delivered via the cannula using air ( 8 bar) after 10 minutes of baseline scanning in a pharmacological (ph)MRI design. This concentration of histamine was chosen as the optimal dose after a pilot study in $3 \mathrm{HCs}$ and $2 \mathrm{AR}$ patient where this dose of histamine resulted in a reduction of $20 \%$ in the peak nasal inspiratory flow (PNIF). Patients did not have the urge to sneeze at this concentration and hence, interfering with the (ph)MRI read-out, as was the case for the dose of $32 \mathrm{mg} / \mathrm{mL}$.

PNIF values were used for measuring nasal flow at baseline and after the nasal provocation at the end of the phMRI scan, as recommended. ${ }^{7}$ For each PNIF measurement, we repeated the PNIF at 3 times with a short interval of 30 seconds and with less than $10 \%$ variation in measured values. The highest PNIF value of 3 values was chosen, irrespective of control or patients. A marginal linear mixed model, more specifically a 2 (substance, histamine vs. saline, within-subject) $\times 2$ (time, pre- versus poststimulation, within-subject) $\times 2$ (group, AR versus HC, between-subject) ANOVA, was performed.

phMRI data were preprocessed and analyzed as described previously. ${ }^{8,9}$ The effect of interest for the present study was the group (AR patients versus $\mathrm{HC}$ )-by-substance (histamine versus saline)by-time interaction effect, comparing the time course of the brain response to histamine vs saline provocation between AR patients and controls. A whole-brain voxel-wise FWE-corrected threshold of 


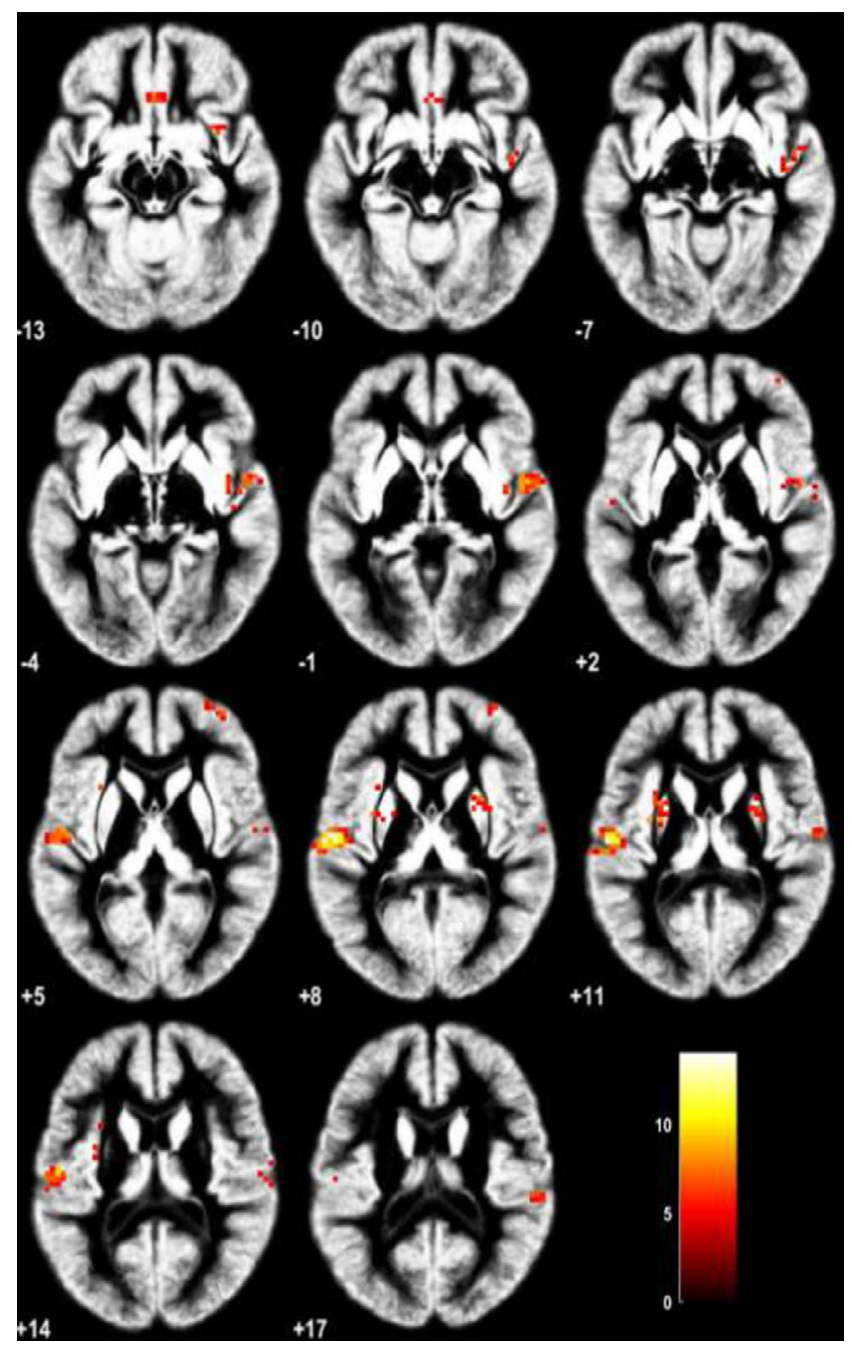

FIGURE 1 Overview of selected brain regions in which the response to histamine (versus saline as a control condition) differs between allergic rhinitis patients and healthy controls. Montage of axial slices overlaid on the average gray matter image of all study subjects. All local maxima pFWE $<0.001$, all clusters $\mathrm{pFWE}<0.001$. The $z$-coordinates of the axial slices were chosen for visualization purposes to show a maximal number of the clusters in Table 1, not to reflect the local maximum of each cluster which is listed in Table 1.The color bar indicates F-values, and numbers indicate $\mathrm{MNI}$ coordinates in the $\mathrm{z}$-direction

$P<.05$ was used combined with an extent threshold of $k=10$ voxels (corresponding to $\mathrm{p}_{\mathrm{FWE}}<0.001$ at cluster level).

In total, 8 HDM AR patients ( 5 females and 3 males) and $7 \mathrm{HC}$ ( 5 females and 2 males) were recruited with a mean age of $22.5 \pm 0.72$ and $23.8 \pm 1.11$ years, respectively. One female HDM AR and two female $\mathrm{HC}$ were excluded due to excessive head movement during MR scanning.

The analysis on PNIF values showed a significant substanceby-time (pre- to postprovocation) interaction effect $(F(1,11)=28.8$, $P=.0002)$, driven by a significant decrease in PNIF after histamine $\left(-47.30 \pm 8.87, \mathrm{p}_{\text {Holm }}=0.0004\right)$, but not after saline $(-5.81 \pm 5.96$, $\mathrm{p}_{\text {Holm }}=0.35$ ) in the entire sample (Supplementary Figure S1). No significant group-by-substance-by-time interaction effect was found $(F(1,11)=0.09, P=.78)$ indicating that the decrease from baseline after histamine compared to saline did not differ between $A R$ and $H C$, with a significant decrease from baseline after histamine but not saline in both groups $(P=.002$ and $P=.015$, respectively). Besides being considered as one the key mediators of allergic inflammation, histamine plays a major role in the regulation of autonomic functions, including energy balance, sleep, and the regulation of body temperature. In addition, histamine is an important mediator involved in nasal itch and in the diagnosis of nasal hyperreactivity, possibly attributing to the effects observed in $\mathrm{HC}$ here.

Brain regions showing a differential response to histamine versus saline in AR patients versus HCs included bilateral mid-/posterior insula, right anterior insula, bilateral postcentral/superior temporal gyrus/rolandic operculum (including secondary somatosensory cortex), bilateral putamen, left cerebellum (crus 1 and 2), right mid-occipital gyrus, bilateral medial orbital gyrus/gyrus rectus, and right middle/superior frontal gyrus (ventrolateral prefrontal cortex) (Table 1, Figure 1). Most of these differential responses were due to stronger activation in HCs vs AR patients, except for the right anterior insula, right middle occipital gyrus, right middle/superior frontal gyrus, and left cerebellum, where a stronger activation was observed in AR patients.

Our pilot study shows for the first time that, besides known differences in local mucosal responses, brain responses to nasal histamine provocation also differ between HDM AR patients versus HCs. More specifically, we found differences in regions that are known to be broadly involved in interoception (ie, monitoring and perceiving the physiological state of the body) ${ }^{10,11}$ and in itch perception, ${ }^{12}$ implicating toward both the perception of symptoms and their putative emotional impact and allergic disease and health.

Our proof-of-concept study holds some limitations: firstly, the small number of participants; secondly, the mild disease severity of AR patients limits our understanding of histamine-activated brain regions in patients with severe AR; and thirdly, differences in brain activity measured with fMRI does not allow to distinguish whether this difference is due to differential activation of histamine receptors in the brain or to differences in afferent signals from the nose or their processing at the brain level. Molecular imaging studies based on positron emission tomography with tracers for the histamine system would be needed to verify the presence of different histamine receptors (ie, $\mathrm{H} 1 \mathrm{R}, \mathrm{H} 2 \mathrm{R}$, and $\mathrm{H} 4 \mathrm{R}$ ).

In conclusion, our results show for the first time activation of different brain regions upon nasal histamine provocation in AR patients versus HC. Further research in a larger group of patients might be useful to investigate brain responses in severe AR patients and to better understand the psycho-emotional impact of chronic allergic inflammation.

\section{CONFLICT OF INTEREST}

The authors declare no conflict of interest. 
Ina Callebaut ${ }^{1}$ Brecht Steelant ${ }^{1}$ iD

Wout Backaert ${ }^{1}$ Ronald Peeters ${ }^{2,3}$ Stefan Sunaert ${ }^{2,3}$ Lukas Van Oudenhove $e^{4,5}$ Peter W Hellings ${ }^{1}$

${ }^{1} \mathrm{KU}$ Leuven Department of Microbiology, Immunology and Transplantation, Allergy and Clinical Immunology Research Group, Leuven, Belgium

${ }^{2}$ Department of Imaging \& Pathology, KU Leuven, Leuven, Belgium

${ }^{3}$ Department of Radiology, University Hospitals Leuven, Leuven, Belgium

${ }^{4}$ Laboratory for Brain-Gut Axis Studies (LaBGAS), Department of Chronic Diseases, Metabolism, and Ageing (CHROMETA), Translational Research Center for Gastrointestinal Disorders (TARGID), University of Leuven, Belgium

${ }^{5}$ Cognitive and Affective Neuroscience Laboratory (CANlab), Center for Cognitive Neuroscience, Department of Psychological and Brain Sciences, Dartmouth College, Hanover, NH, USA

Correspondence

Peter W. Hellings, KU Leuven Department of Microbiology, Immunology and Transplantation, Allergy and Clinical Immunology Research Group, Herestraat 49, 3000 Leuven,

Belgium.

Email: Peter.Hellings@med.kuleuven.be

Van Oudenhove and Hellings joined senior authorship.

\section{REFERENCES}

1. Van Gerven L, Boeckxstaens G, Hellings P. Up-date on neuro-immune mechanisms involved in allergic and non-allergic rhinitis. Rhinology. 2012;50(3):227-235.

2. Callebaut I, De Vries A, Steelant B, et al. Nasal allergen deposition leads to conjunctival mast cell degranulation in allergic rhinoconjunctivitis. Am J Rhinol Allergy. 2014;28(4):290-296.

3. Hsieh JC, Hagermark O, Stahle-Backdahl M, et al. Urge to scratch represented in the human cerebral cortex during itch. J Neurophysiol. 1994;72(6):3004-3008.

4. Pfaar O, Raap U, Holz M, Hormann K, Klimek L. Pathophysiology of itching and sneezing in allergic rhinitis. Swiss Med Wkly 2009;139(3-4):35-40

5. Bensafi M, lannilli E, Poncelet J, et al. Dissociated representations of pleasant and unpleasant olfacto-trigeminal mixtures: an FMRI study. PLoS One 2012;7(6).

6. Rosenkranz MA, Busse WW, Johnstone T, et al. Neural circuitry underlying the interaction between emotion and asthma symptom exacerbation. Proc Natl Acad Sci USA 2005;102(37):13319-13324.

7. Rimmer J, Hellings $P$, Lund VJ, et al. European position paper on diagnostic tools in rhinology. Rhinology. 2019;57(Suppl S28):1-41.

8. Zhao D, Meyer-Gerspach AC, Deloose E. The motilin agonist erythromycin increases hunger by modulating homeostatic and hedonic brain circuits in healthy women: a randomized, placebo-controlled study. Sci Rep. 2018;8(1):1819.

9. Van Oudenhove L, McKie S, Lassman D, et al. Fatty acid-induced gut-brain signaling attenuates neural and behavioral effects of sad emotion in humans. J Clin Investig 2011:121(8):3094-3099.

10. Quadt L, Critchley HD, Garfinkel SN. The neurobiology of interoception in health and disease. Ann N Y Acad Sci 2018;1428(1):112-128.

11. Schulz SM. Neural correlates of heart-focused interoception: a functional magnetic resonance imaging meta-analysis. Philosophical transactions of the Royal Society of London Series B. Biological sciences. 2016;371(1708):20160018.

12. Roberts CA, Giesbrecht T, Stancak A, Fallon N, Thomas A, Kirkham TC. Where Is Itch Represented in the Brain, and How Does it Differ from Pain? An Activation Likelihood Estimation Meta-Analysis of Experimentally-Induced Itch. J Invest Dermatol 2019;139(10):2245-2248.

\section{SUPPORTING INFORMATION}

Additional supporting information may be found online in the Supporting Information section.

\section{Hypersensitivity reactions to platinum-based compounds in the context of pressurized intraperitoneal aerosol chemotherapy (PIPAC): Description and management}

To the Editor,

Pressurized intraperitoneal aerosol chemotherapy (PIPAC) consists of intraperitoneal administration of aerosol platinum salt (PS) chemotherapy under general anesthesia, oxaliplatin (PIPAC-Ox)

IRB permission number: ICM-BCB 2019/10 mostly in the case of colorectal cancer, and cisplatin with doxorubicin (PIPAC-CD) in the case of ovarian and other digestive cancers (Repository text). It can also be alternated with intravenous (IV) chemotherapy. ${ }^{1}$ Systemic toxicity is low, with almost no kidney or liver toxicity. ${ }^{2}$ It is increasingly used. ${ }^{3}$ Only one study described four patients with PS IgE-mediated allergy to PIPAC. ${ }^{4}$ 